\title{
Signatures of nematic quantum critical fluctuations in the Raman spectra of lightly doped cuprates
}

\author{
S. Caprara, ${ }^{1,2}$ M. Colonna, ${ }^{1}$ C. Di Castro, ${ }^{1,2}$ R. Hackl,${ }^{3}$ B. Muschler,${ }^{3}$ L. Tassini,${ }^{3}$ and M. Grilli ${ }^{1,2}$ \\ ${ }^{1}$ Dipartimento di Fisica, Università di Roma Sapienza, Piazzale Aldo Moro 5, I-00185 Roma, Italy \\ ${ }^{2}$ Istituto dei Sistemi Complessi CNR and CNISM Unità di Roma Sapienza \\ ${ }^{3}$ Walther Meissner Institut, Bayerische Akademie der Wissenschaften, 85748 Garching, Germany
}

(Dated: May 30, 2022)

\begin{abstract}
We consider the lightly doped cuprates $\mathrm{Y}_{0.97} \mathrm{Ca}_{0.03} \mathrm{BaCuO}_{6.05}$ and $\mathrm{La}_{2-x} \mathrm{Sr}_{x} \mathrm{CuO}_{4}$ (with $x=$ $0.02,0.04)$, where the presence of a fluctuating nematic state has often been proposed as a precursor of the stripe (or, more generically, charge-density wave) phase, which sets in at higher doping. We phenomenologically assume a quantum critical character for the longitudinal and transverse nematic, and for the charge-ordering fluctuations, and investigate the effects of these fluctuations in Raman spectra. We find that the longitudinal nematic fluctuations peaked at zero transferred momentum account well for the anomalous Raman absorption observed in these systems in the $B_{2 g}$ channel, while the absence of such effect in the $B_{1 g}$ channel may be due to the overall suppression of Raman response at low frequencies, associated with the pseudogap. While in $\mathrm{Y}_{0.97} \mathrm{Ca}_{0.03} \mathrm{BaCuO}_{6.05}$ the low-frequency lineshape is fully accounted by longitudinal nematic collective modes alone, in $\mathrm{La}_{2-x} \mathrm{Sr}_{x} \mathrm{CuO}_{4}$ also charge-ordering modes with finite characteristic wavevector are needed to reproduce the shoulders observed in the Raman response. This different involvement of the nearly critical modes in the two materials suggests a different evolution of the nematic state at very low doping into the nearly charge-ordered state at higher doping.
\end{abstract}

PACS numbers: 74.72.-h, 74.25.nd, 75.25.Dk, 74.40.Kb

\section{INTRODUCTION}

Growing experimental and theoretical evidence indicates that (stripe-like) charge ordering (CO) [1] 3], possibly related to a hidden charge-density-wave quantum critical point near optimal doping [4], plays a role in determining the unconventional properties of superconducting cuprates. Charge ordered textures were assessed by neutron scattering experiments in La cuprates, codoped with Nd [9 11, Ba [12, or Eu [13, and confirmed also by soft resonant x-ray scattering [14 15]. The occurrence of stripe-like charge- and spin-density waves in other cuprates is supported by the similarities of the non-codoped and codoped La cuprates in the spin channel, e.g., the doping dependence of the low-energy incommensurability [16], and the high-energy magnon spectra in $\mathrm{La}_{2-x} \mathrm{Ba}_{x} \mathrm{CuO}_{4}$ [17, $\mathrm{La}_{2-x} \mathrm{Sr}_{x} \mathrm{CuO}_{4}$ (LSCO) [18], and $\mathrm{YBaCuO}_{6+p}$ 1920. These features are well described in terms of striped ground states 21-23. CO in cuprates, possibly with fluctuating character, was also confirmed by EXAFS [24], NMR experiments [25|26], scanning tunneling spectroscopy [27 29], and resonant x-ray measurements 30 33. A recent theoretical analysis of Raman spectra in LSCO [34 showed that nearly critical spin and charge fluctuations coexist at intermediate and high doping. This coexistence also accounts [35] for the specific momentum, energy and doping dependence of the single-particle anomalies, the so-called kinks and waterfalls, observed in photoemission spectra [36].

The above facts, support the occurrence and relevance of (fluctuating) stripes in cuprates and raise the question about their precursors at very low doping [37]. The experimental evidence of rotational symmetry breaking
20, 38,41 points towards nematic order, although it is not yet clear whether this order arises from a melted stripe state [42, from incipient unidirectional fluctuating stripes [43, or from an unrelated $d$-wave-type nematic order which preserves translational symmetry [44]. On the theoretical side, it was recently proposed that a ferronematic state occurs at very low doping, formed by stripe segments without positional order [45]. These segments are oriented because they sustain a vortex and an antivortex of the antiferromagnetic order at their extremes, and break rotational and inversion symmetry. This phase has no order in the charge sector, but induces incommensurate peaks in excellent agreement with experiments in LSCO [46. Recent Monte Carlo calculations 47] showed that, lowering the temperature, the ferronematic state turns into a ferrosmectic state, where the segments have a typical lateral distance $\ell_{c}$, corresponding to $\mathrm{CO}$ with a characteristic wavevector $\mathbf{q}_{c}$ (with $\left|\mathbf{q}_{c}\right| \sim 1 / \ell_{c}$ ). The segments thus appear as the natural precursors of stripes.

It is therefore important to assess nematic order in cuprates. The aim of the present work is to identify the signatures of nematic fluctuations in Raman scattering. This is a bulk (nearly surface-insensitive) probe and measures a response function analogous to that of optical conductivity [48]. However, while the latter averages over the Brillouin zone (BZ), different polarizations of the incoming and outgoing photons weight different parts of the BZ in Raman scattering [49, introducing specific form factors. It turns out that the socalled $B_{1 g}$ and $B_{2 g}$ channels are the most relevant to extract the contributions of collective modes (CMs) in cuprates. We already investigated how these form factors can be exploited to identify the contributions of dif- 
ferent (e.g., charge and spin) critical CMs, based on their different finite wavevectors $34[50 / 53,55]$. There are two classes of CM contributions. In one class, the CMs dress the fermion quasiparticles, introducing self-energy and vertex corrections, which affect the Raman spectra up to substantial fractions of $\mathrm{eV}$ 345354. In the other class, the excitation of pairs of CMs [50], reminiscent of the Aslamazov-Larkin (AL) paraconductive fluctuations near the metal-superconductor transition (see Fig.11), affects mainly the low frequency part of the spectrum and produces an anomalous absorption up to few hundreds of $\mathrm{cm}^{-1}$, as indeed observed, e.g., in LSCO [56]. The analysis for LSCO [50] was based on $\mathrm{CO}$ CMs with finite wavevector $\mathbf{q}_{c}$, while the role of spin CMs was ruled out by symmetry arguments. At moderate doping, the value $\mathbf{q}_{c} \approx( \pm \pi / 2,0),(0, \pm \pi / 2)$ was deduced from inelastic neutron scattering as the double of the wavevector of spin incommensuration [16], within the stripe scheme (we use hereafter a square unit cell on the $\mathrm{CuO}_{2}$ planes, with lattice spacing $a=1$ ). By symmetry arguments, and in agreement with experiments, fluctuations with such $\mathbf{q}_{c}$ give rise to an anomalous absorption in the $B_{1 g}$ channel only. A rotated $\mathbf{q}_{c} \approx 2 \pi( \pm 2 x, \pm 2 x)$ occurs for $x<0.05$, [46], making the anomalous Raman absorption show in the $B_{2 g}$ channel only, consistent with the experiments. However, a similar anomalous absorption in the $B_{2 g}$ channel is observed in $\mathrm{Y}_{1-y} \mathrm{Ca}_{y} \mathrm{Ba}_{2} \mathrm{Cu}_{3} \mathrm{O}_{6+x}$ (YBCO) for doping $p(x, y)$ between 0.01 and 0.06 [52]. Recent measurements 38 do not support the rotation of the spin modulation vector in $\mathrm{YBCO}$, at least down to $p=0.05$, and the extrapolation of the available data indicates that spin incommensuration disappears for $p \approx 0.02-0.03$, while CO seems to disappear for $p<0.08$ [33]. Thus, if only $\mathrm{CO}$ fluctuations were to play a role, the anomalous peak observed in YBCO in the $B_{2 g}$ channel would be unexplained. Furthermore, CO CMs yield in LSCO spectra that are fully satisfactory at $x=0.1$, but less convincing at $x=0.02$, where the experimental lineshape seems to have a composite character, with a main peak accompanied by a shoulder at slightly higher frequencies. This suggests the presence of two CMs contributing to the anomalous absorption in the $B_{2 g}$ channel at low doping in LSCO and raises the question about the nature of the additional CM. The uncertain situation with YBCO and the compositeness of the LSCO spectra call for a critical revision of the results of Ref. [50].

The above mentioned evidences for nematic order make it natural to inquire whether the anomalous Raman absorption observed in underdoped cuprates might be due to nematic fluctuations (not considered in Ref. [50]), possibly mixed with CO fluctuations (in LSCO). Therefore, within the same formal scheme of Ref. [50], we include here the contribution of nematic fluctuations. We find indeed that at low doping the observed anomalous absorption can be due to the excitation of long-wavelength overdamped nematic fluctuations with longitudinal character, whose strong dynamics is apt to reproduce the observed lineshape. While in strongly underdoped YBCO this is enough, in LSCO a secondary CM with finite characteristic wavevector, which we identify with the CO CM, is needed to better represent the lineshape. The doping dependence of the lineshape in LSCO indicates that there is an evolution from a dominating NCM towards a major relevance of the $\mathrm{CO} \mathrm{CM}$, upon increasing doping.

The scheme of the paper is the following: In Sec.II we introduce a phenomenological model of fermion quasiparticles coupled to nearly critical CO CMs and NCMs in underdoped cuprates. Then, we proceed with the theoretical calculation of the Raman response due to these CMs (Secs.II A, IIB, and II C). In Sec.III, we compare the theoretical results with available Raman spectra for underdoped YBCO and LSCO. Sec.IV contains our final remarks and conclusions. Appendix A contains some details about the calculations of the Feynman diagrams involved in the anomalous Raman response. Details of the fitting procedure are found in Appendix B, while a discussion on the role of the pseudogap in the fermionic spectrum is found in Appendix C.

\section{THE FERMION-COLLECTIVE MODE MODEL AND THE RAMAN RESPONSE}

\section{A. The fermion-collective mode model}

We consider a phenomenological model where, similarly to the electron-phonon coupling, electrons are coupled to NCMs or CO CMs. This approach relies on the presence of fermion quasiparticles. This assumption, which is natural in the metallic phase of cuprates, is still justified in the strongly underdoped phase, where angle resolved photoemission [57 and 58] and transport experiments [59 and 60 highlight the presence of fermionic low-energy states (the so-called Fermi arcs) with a substantial mobility, indicating that fermion quasiparticles still survive in this "difficult habitat". Thus, we adopt the Hamiltonian

$$
\mathcal{H}=\sum_{\mathbf{k}, \sigma} \xi_{\mathbf{k}} c_{\mathbf{k} \sigma}^{\dagger} c_{\mathbf{k} \sigma}+\sum_{\mathbf{k}, \mathbf{q}, \sigma} \sum_{\lambda} g_{\lambda}(\mathbf{k}, \mathbf{q}) c_{\mathbf{k}+\mathbf{q} \sigma}^{\dagger} c_{\mathbf{k} \sigma} \Phi_{-\mathbf{q}}^{\lambda}
$$

where $c_{\mathbf{k} \sigma}^{\dagger}\left(c_{\mathbf{k} \sigma}\right)$ creates (annihilates) a fermion quasiparticle with momentum $\mathbf{k}$ and spin projection $\sigma$, and $\xi_{\mathbf{k}}$ is the fermion dispersion on the $\mathrm{CuO}_{2}$ planes of LSCO or YBCO (measured with respect to the chemical potential). Its specific form is rather immaterial for our analysis, once the generic shape of the Fermi surface of cuprates is taken into account. The index $\lambda$ labels transverse $(\lambda=t)$ or longitudinal $(\lambda=\ell)$ nematic fluctuations 6162, and charge fluctuations $(\lambda=c)$, represented by the boson fields $\Phi^{\lambda}$. The quasiparticles couple to NCMs via $g_{\lambda}(\mathbf{k}, \mathbf{q}) \equiv g_{\lambda} d_{\mathbf{k}, \mathbf{q}}^{\lambda}$, with $d_{\mathbf{k}, \mathbf{q}}^{\ell}=\cos \left(2 \varphi_{\mathbf{k}, \mathbf{q}}\right)$ and $d_{\mathbf{k}, \mathbf{q}}^{t}=\sin \left(2 \varphi_{\mathbf{k}, \mathbf{q}}\right)$, where $\varphi_{\mathbf{k}, \mathbf{q}}$ is the angle between $\mathbf{k}$ and $\mathbf{q}$ (see, e.g., Eqs. (2.3) in Ref. [62]). The CO CM has instead a finite characteristic wavevector $\mathbf{q}_{c}$ and couples to the fermion quasiparticle via a weakly momentum dependent coupling $g_{c}(\mathbf{k}, \mathbf{q}) \approx g_{c}$ (i.e., $d_{\mathbf{k}, \mathbf{q}}^{c} \approx 1$ ). 
We assume that these CMs are near an instability and their propagators take the standard Gaussian form, valid within a Landau-Wilson approach, and already adopted for models of fermion quasiparticles coupled to nearly critical charge 44 and spin 6364 CMs in cuprates. As customary in quantum critical phenomena, different damping processes may lead to different dynamical critical exponents $z$, relating the divergent correlation length $\xi$ and time scale $\tau \propto \xi^{z}$. In the case of the nematic instability a multiscale criticality occurs due to the different dynamics of transverse and longitudinal fluctuations 62 65. The longitudinal fluctuations are Landauoverdamped, decay in particle-hole pairs acquiring a dynamical exponent $z_{\ell}=3$, and their propagator is

$$
D_{\ell}\left(\mathbf{q}, \omega_{n}\right)=-\frac{1}{m_{\ell}+c_{\ell}|\mathbf{q}|^{2}+\left|\omega_{n}\right| /|\mathbf{q}|+\omega_{n}^{2} / \Omega_{\ell}},
$$

where $\omega_{n}$ is a boson Matsubara frequency and wavevectors $\mathbf{q}$ are henceforth assumed dimensionless and measured in units of inverse lattice spacing $a^{-1}$ (when needed, conventional units are restored in our formulas by replacing $\mathbf{q}$ with $a \mathbf{q})$. Apart from the term $\propto \omega_{n}^{2}$, this propagator is the same as that in Eq. (2.14) of Ref. 62. Transverse fluctuations have instead $z_{t}=2$, and their propagator is [see, e.g., Eq. (2.15) in Ref. 62]

$$
D_{t}\left(\mathbf{q}, \omega_{n}\right)=-\frac{1}{m_{t}+c_{t}|\mathbf{q}|^{2}+\left|\omega_{n}\right|+\omega_{n}^{2} /\left(\Omega_{t}|\mathbf{q}|^{2}\right)} .
$$

Both propagators, in the static limit $\left(\omega_{n}=0\right)$, are peaked at $\mathbf{q}=0$. Similarly, the nearly critical CO CM has a dynamical critical index $z_{c}=2$, with propagator [see, e.g., Eq. (1) in Ref. 51 or Eq. (2) in Ref. 34]

$$
D_{c}\left(\mathbf{q}, \omega_{n}\right)=-\frac{1}{m_{c}+c_{c}\left|\mathbf{q}-\mathbf{q}_{c}\right|^{2}+\left|\omega_{n}\right|+\omega_{n}^{2} / \Omega_{c}},
$$

peaked at a finite wavevector $\mathbf{q}_{c}$ (actually, at the whole star of equivalent wavevectors). This circumstance allows to reabsorb a factor $\left|\mathbf{q}_{c}\right|^{2}$ in the definition of the parameter $\Omega_{c}$, and marks the difference with respect to the propagator of the transverse NCMs, Eq. (3). In the doping regime we are considering, $\mathbf{q}_{c}$ is directed along the diagonals of the BZ in LSCO with $x<0.05$ 16. According to the discussion in Sec. II, we consider instead that $\mathrm{CO}$ is absent in YBCO with $p \approx 0.015$.

In Eqs. (2 4), the parameters $c_{\lambda}$ set the curvature at the bottom of the CM dispersions, whereas the parameters $\Omega_{\lambda}$ set high-frequency cutoffs. The low-frequency scales $m_{\lambda}$ are proportional to the inverse squared correlation lengths $\xi_{\lambda}^{-2}$, thus being the relevant parameters that measure the distance from criticality.

\section{B. The fermionic loop in the Raman response}

Our theoretical analysis is based on the calculation of the Raman response represented by the Feynman diagrams of Fig. 1] (more details are given in Appendix A).
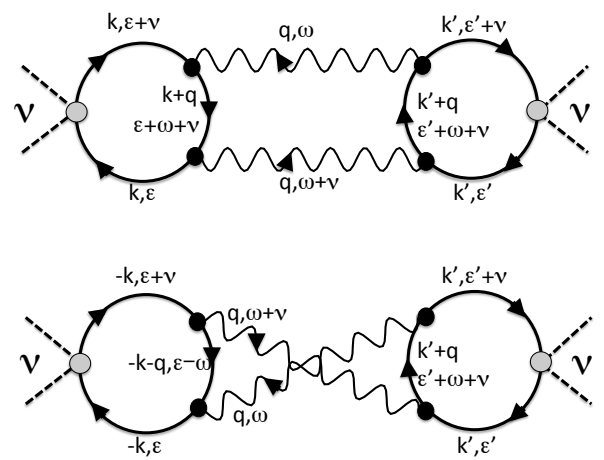

Figure 1. Diagrammatic representation of the Raman response due to the excitation of two CMs. The grey dots represent the $\gamma_{B 1 g}$ or $\gamma_{B 2 g}$ form factors. The solid lines are the propagators of the fermion quasiparticles in the fermionic loops, the wavy lines represent the NCM or CO CM propagators [Eq.s [2 34 4)], which are coupled to the quasiparticles by the coupling functions $g_{\lambda}(\mathbf{k}, \mathbf{q})$ (solid dots).

The first step is to calculate the sum of the fermionic loops with attached direct and crossed boson lines (see top and bottom diagrams in Fig. (1)

$$
\begin{aligned}
& \Lambda_{i}^{\lambda \eta}\left(\mathbf{q}, \nu_{l}, \omega_{m}\right)=T \sum_{\mathbf{k}, n} \gamma_{i}(\mathbf{k}) g_{\lambda}(\mathbf{k}, \mathbf{q}) g_{\eta}(\mathbf{k},-\mathbf{q}) \\
& \times\left[G\left(\mathbf{k}+\mathbf{q}, \epsilon_{n}-\omega_{m}\right)+G\left(\mathbf{k}+\mathbf{q}, \epsilon_{n}+\omega_{m}+\nu_{l}\right)\right] \\
& \times G\left(\mathbf{k}, \epsilon_{n}\right) G\left(\mathbf{k}, \epsilon_{n}+\nu_{l}\right),
\end{aligned}
$$

where $T$ is the temperature, $i=B_{1 g}, B_{2 g}$ labels the form factors, $\gamma_{B 1 g}(\mathbf{k})=\cos \left(k_{y}\right)-\cos \left(k_{x}\right)$ and $\gamma_{B 2 g}(\mathbf{k})=$ $\sin \left(k_{x}\right) \sin \left(k_{y}\right)$ [49], $\nu_{l}$ is the external Matsubara frequency which, once analytically continued, represents the frequency shift between the incoming and the scattered photons, $\omega_{m}$ is the Matsubara frequency of one of the boson propagators in Fig.1 (the other carries $\left.\omega_{m}+\nu_{l}\right), \epsilon_{n}$ is the fermion frequency to be summed over in the fermionic loop, and $G\left(\mathbf{k}, \epsilon_{n}\right)=\left(i \epsilon_{n}-\xi_{\mathbf{k}}\right)^{-1}$ is the fermion quasiparticle propagator. In Eq. (5) we exploited the parity of $G\left(\mathbf{k}, \epsilon_{n}\right), \gamma_{i}(\mathbf{k})$, and $g_{\lambda}(\mathbf{k}, \mathbf{q}) g_{\eta}(\mathbf{k},-\mathbf{q})$ with respect to $\mathbf{k}$.

The dependence of the loop on the CM indexes $\lambda$ and $\eta$ is diagonal: the $\mathrm{CO} \mathrm{CM}$ cannot mix with the NCMs, having a finite characteristic wavevector, and the $\ell$ and $t$ NCMs cannot mix, because the product of $g_{\ell}(\mathbf{k}, \mathbf{q})$ and $g_{t}(\mathbf{k},-\mathbf{q})$, each depending only on the angle between $\mathbf{k}$ and $\mathbf{q}$ and having a different parity, averages to zero when summed with respect to $\mathbf{k}$. This fact entails a selection rule stating that the two NCMs attached to the same fermionic loop must be either longitudinal or transverse. The average over the Fermi surface of two couplings with the same NCM yields a result that is weakly dependent on $\mathbf{q}$ and can be safely approximated to a constant that can be reabsorbed in the definition of the dimensional coupling $g_{\lambda}$. Thus $\Lambda_{i}^{\lambda \eta}\left(\mathbf{q}, \nu_{l}, \omega_{m}\right) \equiv g_{\lambda}^{2} \delta_{\lambda \eta} \Lambda_{i}\left(\mathbf{q}, \nu_{l}, \omega_{m}\right)$. 
Summing over the fermion frequencies, one obtains the general expression

$$
\Lambda_{i}\left(\mathbf{q}, \nu_{l}, \omega_{m}\right)=2 \sum_{\mathbf{k}} \frac{\gamma_{i}(\mathbf{k}) \Delta f_{\mathbf{k}}\left[\Delta \xi_{\mathbf{k}}^{2}-\omega_{m}\left(\omega_{m}+\nu_{l}\right)\right]}{\left(\Delta \xi_{\mathbf{k}}^{2}+\omega_{m}^{2}\right)\left[\Delta \xi_{\mathbf{k}}^{2}+\left(\omega_{m}+\nu_{l}\right)^{2}\right]},
$$

where $\Delta f_{\mathbf{k}} \equiv f\left(\xi_{\mathbf{k}+\mathbf{q}}\right)-f\left(\xi_{\mathbf{k}}\right), \Delta \xi_{\mathbf{k}} \equiv \xi_{\mathbf{k}+\mathbf{q}}-\xi_{\mathbf{k}}$, and $f(z) \equiv\left(\mathrm{e}^{z / T}+1\right)^{-1}$ is the Fermi function.

The next steps are different in the case of NCMs (with characteristic wavevectors $\mathbf{q} \approx 0$ ) and of $\mathrm{CO} \mathrm{CMs}$ (with finite characteristic wavevectors $\mathbf{q}_{c}$ ), and will be dealt with in Sec.IIB 1 and Sec.IIB 2, respectively.

\section{The fermionic loop for NCMs}

To proceed with the calculation of the fermionic loop in the case of NCMs, we consider that the main features of the boson propagators (2) and (3) are their poles at small momenta $q=|\mathbf{q}|$ and even smaller frequencies, because of their dynamics with $z_{\ell}=3\left(\omega \sim q^{3}\right)$ or $z_{t}=2$ $\left(\omega \sim q^{2}\right)$. Thus, expanding the above result for small frequencies and keeping the lowest order in the Matsubara frequencies $\omega_{m}$ and $\omega_{m}+\nu_{l}$, one obtains

$$
\Lambda_{i}\left(\mathbf{q}, \nu_{l}, \omega_{m}\right) \approx 2 \sum_{\mathbf{k}} \frac{\gamma_{i}(\mathbf{k}) \Delta f_{\mathbf{k}}}{\Delta \xi_{\mathbf{k}}^{2}} \approx 2 \sum_{\mathbf{k}} \frac{\gamma_{i}(\mathbf{k})}{\Delta \xi_{\mathbf{k}}} \frac{\partial f\left(\xi_{\mathbf{k}}\right)}{\partial \xi_{\mathbf{k}}}
$$

The summation on $\mathbf{k}$ can be transformed into a twodimensional integral, yielding

$$
\Lambda_{i}(\mathbf{q}) \approx \frac{2 M}{(2 \pi)^{2}} \iint \mathrm{d} k \mathrm{~d} \theta \delta\left(k-k_{F}\right) \frac{\gamma_{i}(k, \theta)}{\Delta \xi_{\mathbf{k}}}
$$

where $\theta$ is the angle between the wavevector $\mathbf{k}$ and the $x$ axis in reciprocal space, and $M$ is the quasiparticle effective mass. By noticing that the form factor $\gamma_{i}(\mathbf{k})$, calculated on the Fermi surface, depends weakly on $k=|\mathbf{k}|$ while it substantially depends on $\theta$, one can write $\gamma_{B 1 g}(k, \theta) \approx \cos (2 \theta) \equiv \gamma_{B 1 g}(\theta)$ and $\gamma_{B 2 g}(k, \theta) \approx$ $\sin (2 \theta) \equiv \gamma_{B 2 g}(\theta)$. When expanding $\Delta \xi_{\mathbf{k}}$ one has to keep track of the inverse band curvature $M$ (otherwise the integral vanishes). The limit $|\mathbf{q}| \rightarrow 0$ can then be taken, and the final result is that the fermionic loop depends only on the angle $\phi$ between $\mathbf{q}$ and the $x$ axis. This dependence can be made explicit observing that the denominator $\Delta \xi_{\mathbf{k}}$ in Eq. (6) depends on the cosine of the angle $\theta-\phi$ between $\mathbf{k}$ and $\mathbf{q}$. Shifting the variable $\theta-\phi \rightarrow \theta$, one is left with $\gamma_{i}(\theta+\phi)$ in the numerator. Expanding, one has $\gamma_{B 1 g}(\theta+\phi)=\gamma_{B 1 g}(\theta) \gamma_{B 1 g}(\phi)-\gamma_{B 2 g}(\theta) \gamma_{B 2 g}(\phi)$ and $\gamma_{B 2 g}(\theta+\phi)=\gamma_{B 2 g}(\theta) \gamma_{B 1 g}(\phi)+\gamma_{B 1 g}(\theta) \gamma_{B 2 g}(\phi)$. The integral with respect to $\theta$ of the terms with $\gamma_{B 2 g}(\theta)$ vanishes by symmetry. Thus, we finally obtain

$$
\Lambda_{i}(\phi) \approx \frac{M^{2}}{\pi k_{F}^{2}} \gamma_{i}(\phi)
$$

This result, which is crucial in our development, implies that the original form factor $\gamma_{i}(\theta)$ coupling the fermion quasiparticles to the incoming and outgoing photons in the Raman vertex, in the integrated form of the loops, is translated into a direct coupling of the photons to the NCMs with the same form factor $\gamma_{i}(\phi)$.

\section{The fermionic loop for the CO CMs}

The fermionic loop for the CO CMs has been calculated in Ref. [50], and we recall here the main results. The main difference with respect to the calculation of Sec.II B 1, is that the propagator (4) is peaked at finite wavevectors $\mathbf{q}_{c}$. Then, the sum over $\mathbf{k}$ in Eq. (5) is now dominated by the neighborhood of the points along the Fermi surface where $\xi_{\mathbf{k}}=\xi_{\mathbf{k}+\mathbf{q}_{c}}$, i.e., the so-called hot spots. The results is

$$
\Lambda_{i}\left(\mathbf{q}_{c}\right) \approx \frac{1}{2 \pi^{2}} \ln \left|\frac{W_{+}}{W_{-}}\right| \sum_{H S} \frac{\gamma_{i, H S}}{v_{H S}^{2} \sin \alpha_{H S}}
$$

where $W_{ \pm}$are the upper and lower cutoffs for the linearized band dispersion at the hot spot, while $\gamma_{i, H S}$ and $v_{H S}$ are, respectively, the Raman form factor and the Fermi velocity at $\mathbf{k}=\mathbf{k}_{H S}$, and $\alpha_{H S}$ is the angle between the Fermi velocities at the two hot spots connected by the given $\mathbf{q}_{c}$. For $\mathbf{q}_{c}$ along high symmetry directions (i.e., the axes and the diagonals) of the BZ, the moduli of the Fermi velocities in $\mathbf{k}_{H S}$ and $\mathbf{k}_{H S}+\mathbf{q}_{c}$ are equal. As pointed out in Ref. [50], summing over $\mathbf{k}$ at fixed $\mathbf{q}_{c}$, various different hot spots are visited, where, due to the above-mentioned symmetry, the form factors can have pairwise equal magnitude and equal or opposite signs. As a consequence, the terms in the above hot-spot summation can add or cancel each other. This induces a "selection rule" which, in the case pertinent to the strongly underdoped LSCO, where $\mathbf{q}_{c}$ is short and directed along the $( \pm 1, \pm 1)$ directions, leads to finite $B_{2 g}$ vertex loops, while the $B_{1 g}$ vertex loops vanish by symmetry.

\section{The Raman response}

Few considerations are now in order. First of all, the NCM propagators, Eqs. (2) and (3), do not depend on the angle $\phi$ and therefore the product of the two fermionic loops entering the diagrams of Fig. 1 only introduces a multiplicative constant factor, which can be enclosed in the overall intensity of the Raman response. However, we emphasize that the $\phi$ integration, to be performed when the summation over $\mathbf{q}$ is carried out, introduces an important selection rule: The fermionic loops with attached Raman vertices, enter pairwise in the response diagrams of Fig. 1 and must both be of the same symmetry, $B_{1 g}$ or $B_{2 g}$. Similarly, the CO propagator 4 depends only on the magnitude of the deviation of $\mathbf{q}$ from $\mathbf{q}_{c}$. In this case the Raman response is given by a first summation on all the possible $\mathbf{q}_{c}$ of $\left[\Lambda_{i}\left(\mathbf{q}_{c}\right)\right]^{2}$ and an internal integral over $\left|\mathbf{q}-\mathbf{q}_{c}\right|$ of two CO CM propagators. 
Thus, both for the two nematic CMs and for the $\mathrm{CO}$ $\mathrm{CM}$, the sum of the two diagrams of Fig.1 1 reads

$$
\chi_{i, \lambda}\left(\nu_{l}\right)=K_{i, \lambda} T \sum_{n} \int_{0}^{\bar{q}} \mathrm{~d} q \frac{q}{\bar{q}^{2}} D_{\lambda}\left(q, \omega_{n}\right) D_{\lambda}\left(q, \omega_{n}+\nu_{l}\right),
$$

where $\bar{q} \sim 1$ is the momentum cutoff, its precise value being re-absorbable in a multiplicative rescaling of the parameters of the $\mathrm{CM}$ propagator, $q=|\mathbf{q}|$ for the NCMs, and $q=\left|\mathbf{q}-\mathbf{q}_{c}\right|$ for the CO CMs. The factor $K_{i, \lambda}$ comes from the product of two fermionic loops and is proportional to $g_{\lambda}^{4}$ (each loop $\Lambda_{i}$ carrying two fermion-CM coupling constants). For NCMs, $K_{i, \lambda} \equiv$ $M^{4} g_{\lambda}^{4}\left\langle\left[\gamma_{i}(\phi)\right]^{2}\right\rangle /\left(\pi k_{F}^{2}\right)^{2}$, with $\lambda=\ell$ or $t$, and $\left\langle\left[\gamma_{i}(\phi)\right]^{2}\right\rangle$ is the angular average of the square of the function $\gamma_{i}(\phi)$ that appears in Eq. (7), whereas for the CO CM we have [cf. Eq. (8)] $K_{i, c}=g_{c}^{4} \sum_{\mathbf{q}_{c}}\left[\Lambda_{i}\left(\mathbf{q}_{c}\right)\right]^{2}$, that vanishes in the $B_{1 g}\left(B_{2 g}\right)$ channel for diagonal (vertical/horizontal) $\mathbf{q}_{c}$. This "selection rule" is the only place where the finite wavevector of the CO CM plays a role within our nearly critical theory of Raman absorption. This selection rule is instead absent in the case of the NCMs, which are peaked at $\mathbf{q}=0$.

The analytic continuation to real frequencies $i \nu_{l} \rightarrow$ $\omega+i \delta$ and the use of the spectral representation of the boson propagators finally yield the Raman response

$$
\begin{aligned}
\chi_{i, \lambda}^{\prime \prime}(\omega) & =A_{i, \lambda} \int_{-\infty}^{+\infty} \mathrm{d} z\left[b\left(z_{-}\right)-b\left(z_{+}\right)\right] \int_{0}^{1} \mathrm{~d} q \\
& \times 2 q F_{\lambda}\left(z_{+}, q\right) F_{\lambda}\left(z_{-}, q\right),
\end{aligned}
$$

where $b(z) \equiv\left(\mathrm{e}^{z / T}-1\right)^{-1}$ is the Bose function, and we performed the customary symmetrization $z \rightarrow z-\frac{\omega}{2} \equiv$ $z_{-}$and $z+\omega \rightarrow z+\frac{\omega}{2} \equiv z_{+}$, which makes explicit the fact that Eq. (9) is an odd function of $\omega$. The constant multiplicative prefactors, including those transforming the Raman susceptibility into the measured Raman response, are reabsorbed in the parameters $A_{i, \lambda}$. Unfortunately a fully analytical expression for $A_{i, \lambda}$ cannot be given, the Raman response being affected by resonance effects that prevent even order-of-magnitude estimates. However, whenever we studied the contribution of critical $\mathrm{CMs}$ in situations where the prefactors can be explicitly calculated, like optical conductivity 34|51, or angle resolved photoemission spectra [35], we always found that the dimensionless coupling constants are of order one, in a regime of moderate coupling.

The spectral density of the longitudinal NCMs is

$$
F_{\ell}(z, q)=\frac{\frac{z}{q}}{\left(m_{\ell}+c_{\ell} q^{2}-\frac{z^{2}}{\Omega_{\ell}}\right)^{2}+\frac{z^{2}}{q^{2}}},
$$

while the spectral density of the transverse NCMs is

$$
F_{t}(z, q)=\frac{z}{\left(m_{t}+c_{t} q^{2}-\frac{z^{2}}{\Omega_{t} q^{2}}\right)^{2}+z^{2}} .
$$

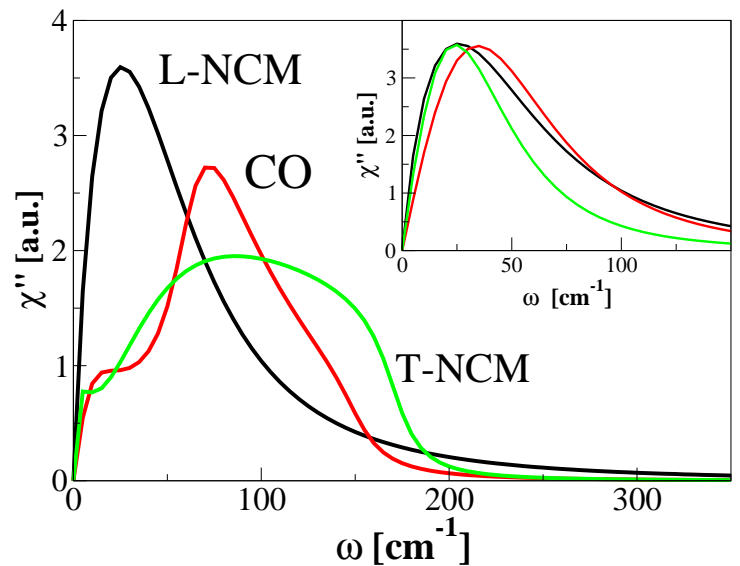

Figure 2. Schematic representation of the AL-like Raman response of the three different CMs: longitudinal L-NCM (black curve), transverse (T) NCM (green curve), and CO CM (red curve). Inset: AL-like Raman response from different CMs, but taken with the same nearly critical set of parameters $\left(m_{\lambda}=17 \mathrm{~cm}^{-1}, \Omega_{\lambda}=70 \mathrm{~cm}^{-1}, c_{\lambda}=3.16\right)$ The amplitudes are instead rescaled by factors of order one to bring all responses to a common maximal height for easier comparison. The coloring of the lines is the same as in the main panel.

Finally, for the CO CMs we find

$$
F_{c}(z, q)=\frac{z}{\left(m_{c}+c_{c} q^{2}-\frac{z^{2}}{\Omega_{c}}\right)^{2}+z^{2}} .
$$

The anomalous peak of the Raman response, both in LSCO and YBCO, is strongly temperature dependent, it shrinks and softens upon reducing $T$. This behavior is naturally encoded in the temperature dependence of the mass $m_{\lambda}$ of the CMs. In general, the low-frequency scale $m_{\lambda}$ controls the slope of the Raman response, while the scales $\omega_{1} \sim \sqrt{m_{\lambda} \Omega_{\lambda}}$ and $\omega_{2} \sim \sqrt{\left(m_{\lambda}+c_{\lambda}\right) \Omega_{\lambda}}$ set the frequency window over which the spectral function of the corresponding CM is sizable. However, the different dynamical properties and values of the parameters of the CMs mirror into different shapes of the AL-like Raman responses, which are schematically represented in Fig. 2. We point out that the curves displayed in this panel do not exhaust all the possible regimes of parameters, and only represent the corresponding $\mathrm{CM}$ in the regime where, after a thorough analysis, they were found to better reproduce the various features of the Raman response. The inset of the same figure reports instead the behavior of Raman absorption spectra (from the AL processes) due to the various CMs. In this inset, while we rescale the height to bring all responses to the same maximal height, we use the same nearly critical set of parameters to highlight the differences arising purely from the different form of the propagators and dynamical critical index $z$. Apparently the shape of the spectra is quite similar, but the behavior upon changing the mass is dif- 
ferent on a quantitative level. In particular we found that the $z=2$ propagators shift the position of the maxima upon reducing $m$ more rapidly than the $\mathrm{NCM} z=3$ propagator. Since we apply a strict fitting protocol (see below in Section III), which fixes all parameters and follows the temperature evolutions of the main peaks by only changing $m$, these different behavior affects in a substantial way the accuracy of the fits. An inspection of Fig. 3 in Ref. 50 shows that the fits with a $z=2 \mathrm{CO}-\mathrm{CM}$ are not very accurate at low temperatures. Instead we will see in the next Section that the $z=3 \mathrm{NCM}$ does a much better job within the adopted strict fitting protocol and therefore it will be considered henceforth as the primary (i.e., most critical) CM. The additional shoulder in the spectra of LSCO, is instead better reproduced by the CO curve in Fig. 2 than by the broader T-NCM curve, when both CMs are taken in the regime of parameters apt to describe this spectral feature. Therefore at these doping levels the CO CM acts as the secondary CM in LSCO.

\section{RESULTS}

\section{A. Raman absorption in $\mathbf{Y}_{0.97} \mathbf{C a}_{0.03} \mathbf{B a}_{2} \mathbf{C u}_{3} \mathbf{O}_{6.05}$}

An anomalous Raman absorption at low frequencies, up to few hundreds of $\mathrm{cm}^{-1}$, is experimentally found in the $B_{2 g}$ channel in lightly doped YBCO with $p \leq 0.05$ [52. Since, however, the whole spectra also display broad absorptions up to electronic energy scales, we first extract the specific anomalous low-frequency contributions. To this purpose, we subtract from the low-temperature spectra the spectra obtained at the highest measured temperature. This subtraction is delicate because at temperatures below about $150-200 \mathrm{~K}$, the spectra are characterized by the formation of a pseudogap over a frequency range of several hundreds of $\mathrm{cm}^{-1}$, which reduces the electronic background. Then, the simple subtraction leads to regions of negative absorptions, which are obviously meaningless. In the Appendix B we provide the detailed procedure adopted to circumvent this drawback. In Fig. 3, the data processed according to the previous procedure, are shown for $p \approx 0.015$.

The experimental lineshape clearly resembles the LNCM spectrum in Fig.2, which is narrow due to the $z_{\ell}=3$ damped dynamics of the corresponding CM, whose temperature dependence is ruled by the mass $m_{\ell}$. Indeed, the data in Fig.3 are best fitted with the only contribution of longitudinal NCMs. In the spirit of our nearly-critical approach, we only adjust their mass $m_{\ell}(T)$, while keeping all other parameters (i.e., the highfrequency cutoffs of the CM propagator, the $c_{\ell}$ coefficients, and the overall intensity coefficient $A_{\ell}$ ) fixed at all temperatures. This strict procedure was already successfully adopted in Ref. [50] and seems to us the most suitable to pinpoint the quantum nearly-critical character of the collective excitations responsible for the anomalous Raman absorption. The fits with this restricted

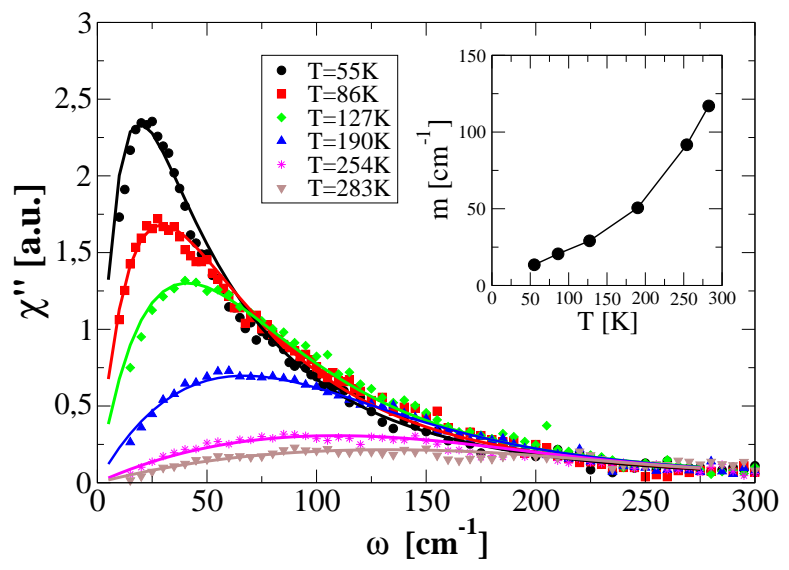

Figure 3. Subtracted experimental Raman absorption spectra in the $B_{2 g}$ channel, at various temperatures, for YBCO at $p \approx 0.015$ (symbols). The theoretical fits (solid lines) consider the contribution of the longitudinal NCM only. The fitting parameters are $c_{\ell}=0.63 \mathrm{~cm}^{-1}, \Omega_{\ell}=110 \mathrm{~cm}^{-1}, A_{\ell}=5.0$ (a.u.). The inset reports the temperature dependence of the mass of the longitudinal NCM (black circles).

procedure turn out to be quite good. Of course they could be further improved if this constrained procedure were relaxed. The fits reproduce well the lineshapes and the strong temperature dependence of the peak, encoded in the rapid decrease of the mass with temperature, as shown in the inset of Fig.3. From this inset, it is evident that $m_{\ell}(T)$ decreases with $T$. Its linear extrapolation starting from high temperature should vanish at some finite critical temperature for the onset of nematicity $(\approx 125 \mathrm{~K})$, if static order would occur. However, at lower temperatures, the mass seems instead to saturate, likely indicating that nematic order stays short-ranged and dynamic.

\section{B. Raman absorption in $\mathrm{La}_{2-x} \mathrm{Sr}_{x} \mathrm{CuO}_{4}$}

Figs. 4 and 5 report the experimental Raman spectra in the $B_{2 g}$ channel, for LSCO samples at doping $x=0.02$ and $x=0.04$ and various temperatures. The raw data were again processed according to the procedure described in the Appendix B. As mentioned above, the anomalous Raman absorption observed in LSCO is characterized by a lineshape that is more complex than in YBCO, and displays a peculiar shoulder or, at low $T$, even a secondary peak, see Fig.5. The anomalous peak and the shoulder (or secondary peak) both depend on temperature, but their frequency and intensity are not simply related by constant multiplicative factors. The shoulder (or secondary peak) becomes stronger with increasing doping. This indicates that the excitations responsible for this absorption have a distinct dynamics. 


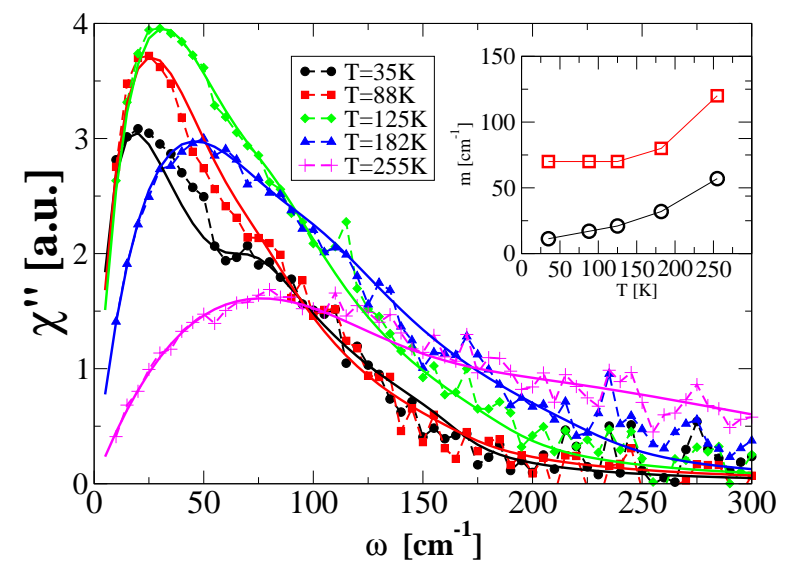

Figure 4. Subtracted experimental Raman absorption spectra in the $B_{2 g}$ channel, at various temperatures, for LSCO at $x=0.02$ (symbols). The theoretical fits (solid lines) consider the contribution of the longitudinal $\mathrm{NCM}$ and of the CO CM, with $c_{\ell}=3.16 \mathrm{~cm}^{-1}, c_{c}=333 \mathrm{~cm}^{-1}, \Omega_{\ell}=70 \mathrm{~cm}^{-1}, A_{\ell}=8.3$ (a.u.). The other fitting parameters are reported in Fig.6 The inset reports the temperature dependence of the mass of the longitudinal NCM (black circles) and of the CO CM (red squares).

Again these absorptions are described by the AL-like processes (direct and crossed, see Fig.11). Owing to the selection rules found in Sec.II. the response due to two (or more) CMs is the sum of the responses associated with each individual CM. As already mentioned, our thorough analysis showed that the primary anomalous absorption should be attributed to the longitudinal NCM, which has the stronger dynamical behavior. Within our context, the transverse NCM and the CO $\mathrm{CM}$ are the two candidates for the shoulder (or secondary peak). Looking at the lineshape of the two CMs reported in Fig.2, it is easy to convince oneself that the best choice for a good fit is the CO CM, due to its much more pronounced peaked form at intermediate frequency. We also attempted a fit with the transverse NCM. At $x=0.02$ we obtained a reasonable fit taking a very large and almost temperature independent CM mass, which is hardly compatible with our assumption of nearly critical CMs. Moreover, at $x=0.04$, when the shoulder evolves into a secondary peak, the attempt failed completely. Thus, we ruled out a contribution of transverse NCMs.

Again, having attributed the main peak to the more critical longitudinal NCM, we describe the low-frequency side of the spectra by only adjusting the mass $m_{\ell}(T)$ of this excitation, while keeping all other parameters of this mode (i.e., the high-frequency cutoff of the CM propgator, the $c_{\ell}$ coefficient, and the overall intensity coefficient $A_{\ell}$ ) fixed at all temperatures, within the temperature range considered here. Thus, we obtain the marked temperature dependence of the longitudinal NCM mass,

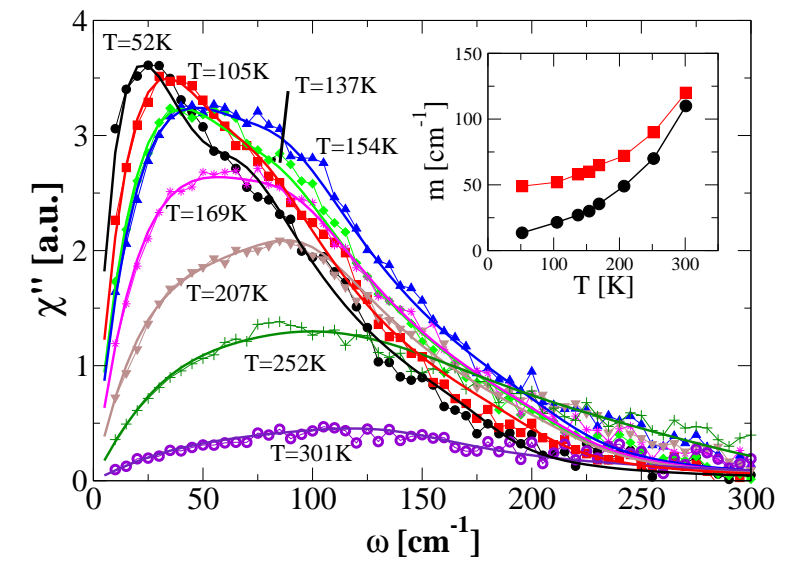

Figure 5. Subtracted experimental Raman absorption spectra in the $B_{2 g}$ channel, at various temperatures, for LSCO at $x=$ 0.04 (symbols). The theoretical fits (solid lines) consider the contribution of the longitudinal NCM and of the CO CM, with $c_{\ell}=3.16 \mathrm{~cm}^{-1}, c_{c}=333 \mathrm{~cm}^{-1}, \Omega_{\ell}=50 \mathrm{~cm}^{-1} . A_{\ell}=7.14$ (a.u.). The other fitting parameters are reported in Fig.6. The inset reports the temperature dependence of the mass of the longitudinal NCM (black circles) and of the CO CM (red squares).

which is reported in the insets of Figs. 4 and 5 . On the other hand, the complete quantitative agreement between data and theoretical fits is only obtainable by adjusting more freely the secondary CO CM. This mode is therefore allowed to vary its parameters with $T$, as reported in Fig.6. The temperature dependence of the $\mathrm{CO}$ CM parameters $\Omega_{c}$ and $A_{c} \propto g_{c}^{4}$ likely reflects an increasing damping and a decreasing coupling to the fermion quasiparticles with increasing $T$. Of course, the estimates and variations of these parameters may be quantitatively affected if the constraint of $T$-independent parameters for the longitudinal NCM (but for its mass $m_{\ell}$ ) were relaxed. Furthermore, we cannot exclude that static nematic order has eventually occurred, e.g., in the sample with $x=0.02$ at the lowest temperature. In this case our analysis, which is only valid above the critical temperature, should be modified to deal with a broken-symmetry phase. This might reflect in a reduction of the primary peak, due to the freezing of NCM fluctuations, and could be the cause of the non monotonic behavior of the peak height as a function of $T$, observed in the sample with $x=0.02$. To asses the occurrence of static nematic order at low temperature, a systematic experimental investigation in this temperature regime is needed.

\section{DISCUSSION AND CONCLUSIONS}

Our analysis showed that the anomalous Raman absorption observed in underdoped cuprates can be inter- 


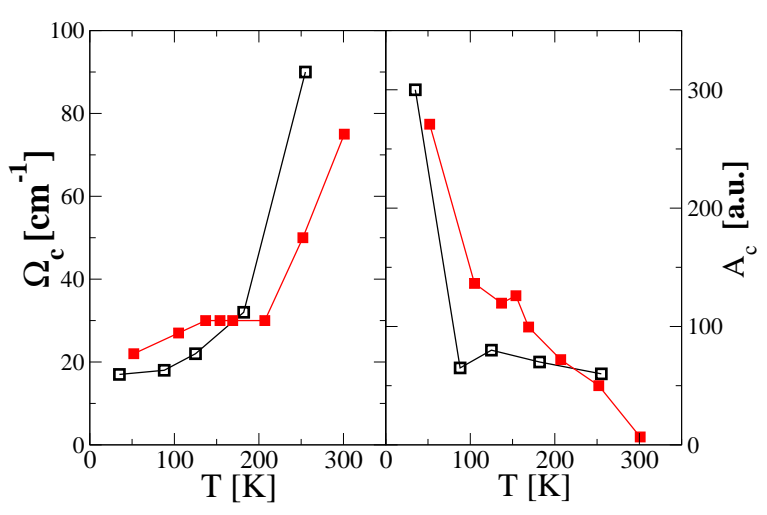

Figure 6. (a) High-frequency cutoff $\Omega_{c}$ for the CO CM for a sample at $x=0.02$ (black empty squares) and at $x=0.04$ (red filled squares). (b) Amplitude coefficients $A_{c}$ for the CO CM for a sample at $x=0.02$ (black empty squares) and at $x=0.04$ (red filled squares).

preted in terms of direct excitation of nearly critical CMs (see Fig.11). The strong temperature dependence of the mass (i.e., inverse square correlation length) of the "primary" CM, identified as the longitudinal NCM (with dynamical critical index, $z_{\ell}=3$ ), captures the correspondingly strong variation of the spectra. This CM alone fully accounts for the spectra of YBCO. In LSCO, instead, a distinct "secondary" CM, with different dynamical critical index $z=2$, is needed to reproduce the composite lineshape. Within the two candidates considered in our scheme (transverse $\mathrm{NCM}$ and $\mathrm{CO} \mathrm{CM}$ ), our fits indicate that the $\mathrm{CO} \mathrm{CM}$ is the most suitable.

For symmetry reasons the secondary $\mathrm{CO} \mathrm{CM}$ cannot occur in all channels: The first two rows in the sketch of Fig. 7 7 summarize the findings of Ref. [50 in LSCO as far as CO is concerned: The correct CO (i.e., with finite $\mathbf{q}_{c}$ in the direction compatible with inelastic neutron scattering experiments) appears as an observed absorption (green case) only in the theoretically predicted channel.

Two questions still remain to be answered, in order to complete the scheme of Fig.7 7 First of all, the NCMs would equally contribute to the $B_{1 g}$ and $B_{2 g}$ channels. Therefore, they would not only add to the CO fluctuations that give absorption in the $B_{1 g}$ channel at larger doping $(x>0.05)$ in LSCO, but would also give rise to absorption in the $B_{2 g}$ channel. Since this is not observed (the corresponding box is red in Fig.77), we infer that NCMs disappear in LSCO at $x>0.05$ (see the comment box in the first row of Fig.77). This is consistent with the observation of an increasingly stronger stripe order at higher doping [50, where $\mathrm{CO} \mathrm{CM}$ alone [along the $(1,0)$ and $(0,1)$ directions of the BZ] accounted for the anomalous Raman absorption at $x=0.10$ and 0.12 .

The second related question is: If the $\mathrm{NCMs}$ are present and contribute to the absorption in $B_{2 g}$ at low doping both in LSCO and YBCO, why are they not vis-

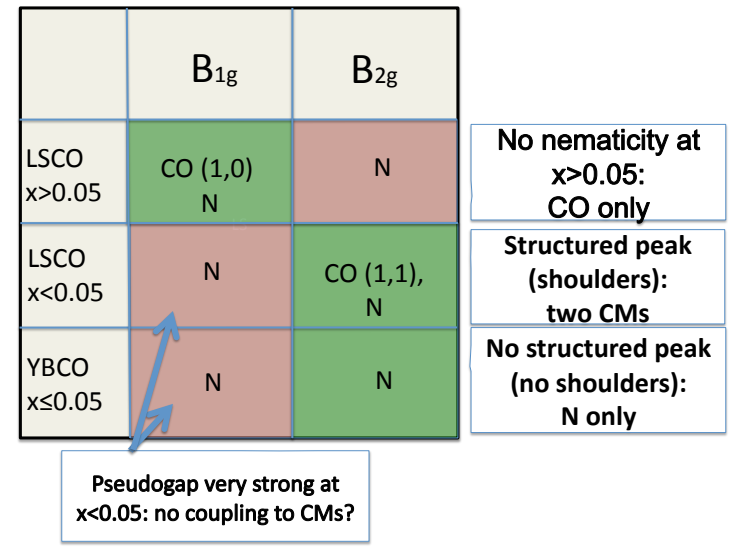

Figure 7. Schematic comparison of the theoretical expectations and the experimental observation of an anomalous Raman absorption. The theoretically involved CMs are indicated with $\mathrm{N}$ in the case of the $\mathrm{NCM}$, while for $\mathrm{CO}$ we also report the direction of the characteristic wavevector, as established by inelastic neutron scattering. The related symbols only appear in the box where they are expected to contribute on the basis of symmetry arguments. The experimental observation of an anomalous Raman absorption is depicted as a green case in the column of the corresponding channel. Red cases indicate, instead the lack of anomalous Raman absorption in experiments. Our remarks and possible indications (in boldface) are contained in the comment boxes.

ible in the (for them allowed) $B_{1 g}$ channel? As yet, we do not have a definite answer. We argue that the strong pseudogap occurring in lightly doped cuprates at $T<200 \mathrm{~K}$ could play a key role in suppressing the $B_{1 g}$ absorption. Specifically, the $B_{1 g}$ form factors select the quasiparticles in the fermionic loops of Fig.1 precisely from the BZ regions where the pseudogap is largest. Therefore, only the quasiparticles in the remaining Fermi arcs, mostly weighted by the $B_{2 g}$ form factors, remain to couple the Raman photons with the NCMs. In Appendix $\mathrm{C}$, we obtained a numerical estimate of this suppression, finding indeed that it can be substantial.

Based on the above discussion, we can draw the schematic "phase diagram" for underdoped cuprates reported in Fig.8. Despite its speculative character, it is compatible with various theoretical and experimental findings, and accounts for the assessed relevance of nematic order in cuprates [20, 38 41]. It also complies with the proposal of a nematic order resulting from the melting of stripes [42] or of a nematic or smectic phase in strongly underdoped LSCO (and possibly YBCO) 47], arising from the aggregation of doped charges in short segments (blue region). The orientation of these segments breaks the lattice $C_{4}$ rotational symmetry preparing the route to $\mathrm{CO}$ at higher doping, when the segments merge into stripes (green region). The fluctuating character of the nematic phase should give rise to nearly critical fluctuations of the form of Eqs. (2) and (3). On 


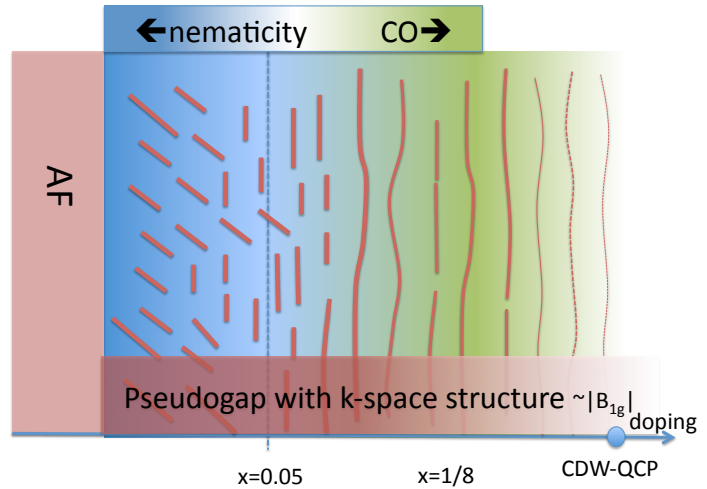

Figure 8. Schematic evolution of the nematic (blue region) and stripe CO (green region) phases in underdoped cuprates with doping (disregarding superconductivity). The pseudogap region, where the Raman response in the $B_{1 g}$ channel is expected to be suppressed, is highlighted in red. Upon increasing doping, the nematic phase evolves into a $\mathrm{CO}$ phase, which in turn vanishes at a $\mathrm{CO}$ quantum critical point around optimal doping. The orientation of the segments and/or stripes may change with cuprate family and doping.

the other hand, CO fluctuations become prominent by increasing doping and appear in the $B_{1 g}$ channel above $x=0.05$. In LSCO these fluctuations are present (in the diagonal directions of the BZ) also at $x<0.05$ and contribute to the $B_{2 g}$ absorption, but the tendency of $\mathrm{CO}$ fluctuations to become more relevant at larger doping is clearly visible by comparing Figs. 4 and 5 . At the same time, the insets of Figs. 4 and 5 also display an increase of the low-temperature limit of the correlation length of the $\mathrm{CO} \mathrm{CM}$ upon increasing doping. Hence, nematic and CO fluctuations coexist in very underdoped LSCO, the predominance shifting from nematic to $\mathrm{CO}$ with increasing doping. This indicates a continuous evolution from the nematic (charge segment) phase to the stripe phase where charge and spin degrees of freedom are tightly bound, yielding a definite relation between spin and charge incommensurabilities (typical of the stripe phase). We relied on this relation to implement our symmetry-based selection rules for LSCO. On the other hand, our finding that NCMs alone are relevant in YBCO at very low doping supports the idea that oriented charge segments may occur in this material as well, accounting for the orderparameter-like disappearance of the incommensurability in the spin response with increasing temperature [45 47], as observed in Refs. [20]38]. The lack of CO fluctuations at low doping and the opposite doping dependence of the charge and spin characteristic wavevectors [33] indicate a nematic-to-CO switching different from that in LSCO. However, both materials seem to eventually evolve into a charge-density-wave phase ending into a quantum critical point around optimal doping, as theoretically proposed [4] and recently observed [33.

\section{ACKNOWLEDGMENTS}

S.C. and M.G. acknowledge financial support form the University of Rome "Sapienza" with the Project Awards n. C26H13KZS9.

\section{Appendix A: Details on the calculation of the Raman response}

Inspection of Fig. 1 1 shows that the diagrammatic structure of the Raman response due to the excitation of two CMs involves two inequivalent fermionic loops (on the left of the two diagrams), which multiply two CM propagators and the fermionic loop on the right of the diagrams. Calling $\Lambda_{i}^{\lambda \eta}\left(\mathbf{q}, \nu_{l}, \omega_{m}\right)$ the sum of the two different fermionic loops (frequencies and momenta are those displayed in Fig. 1), we can write the expression for the sum of the two diagrams as

$$
\begin{aligned}
\chi_{i j}^{\lambda \eta}\left(\nu_{l}\right) & =T \sum_{\mathbf{q}, m} \Lambda_{i}^{\lambda \eta}\left(\mathbf{q}, \nu_{l}, \omega_{m}\right) \\
& \times D_{\lambda}\left(\mathbf{q}, \omega_{m}\right) D_{\eta}\left(\mathbf{q}, \omega_{m}+\nu_{l}\right) L_{j}^{\lambda \eta}\left(\mathbf{q}, \nu_{l}, \omega_{m}\right),
\end{aligned}
$$

where $i, j=B_{1 g}, B_{2 g}, \lambda, \eta=\ell, t, c$, and $L_{j}^{\lambda \eta}\left(\mathbf{q}, \nu_{l}, \omega_{m}\right)$ stands for the fermionic loop in the right part of the diagrams. The above expression can be made symmetric also with respect to the latter fermionic loop, observing that the integrated $\mathbf{k}^{\prime}$ can be changed into $-\mathbf{k}^{\prime}$, and the fermionic Matsubara frequency $\epsilon_{n}^{\prime}$ can be shifted to $\epsilon_{n}^{\prime}-$ $\nu_{l}$ (frequencies and momenta are those displayed in the fermionic loop on the right of both diagrams in Fig.11. Then, exploiting the parity of the CM propagators with respect to both momentum and frequency arguments, we can take $\mathbf{q} \rightarrow-\mathbf{q}$ and $\omega_{m} \rightarrow-\omega_{m}, \nu_{l} \rightarrow-\nu_{l}$. Summing the two equivalent expressions and dividing by 2 we are finally led to calculate

$$
\begin{aligned}
\chi_{i j}^{\lambda \eta}\left(\nu_{l}\right) & =\frac{T}{2} \sum_{\mathbf{q}, m} \Lambda_{i}^{\lambda \eta}\left(\mathbf{q}, \nu_{l}, \omega_{m}\right) \\
& \times D_{\lambda}\left(\mathbf{q}, \omega_{m}\right) D_{\eta}\left(\mathbf{q}, \omega_{m}+\nu_{l}\right) \Lambda_{j}^{\lambda \eta}\left(\mathbf{q}, \nu_{l}, \omega_{m}\right) .
\end{aligned}
$$

This expression has the formal structure of a Raman response where to CMs are directly excited by the scattered electromagnetic radiation, and $\Lambda_{i}^{\lambda \eta}\left(\mathbf{q}, \nu_{l}, \omega_{m}\right)$ plays the role of an effective Raman vertex, resulting from the sum of the fermionic loops with attached direct and crossed boson lines in Fig.11. In Sec. IIB we discuss the calculation of the effective vertex $\Lambda_{i}^{\lambda \eta}\left(\mathbf{q}, \nu_{l}, \omega_{m}\right)$. This calculation is further specialized to the cases of NCMs and $\mathrm{CO}$ CMs in Secs. IIB 1 and IIB 2, respectively.

\section{Appendix B: Fitting procedure of the anomalous Raman absorption}

To fit the anomalous contribution of the Raman absorption due to two virtual CMs, as represented in Fig. 1. 
one needs to subtract the regular part of the spectra arising, e.g., from the dressed quasiparticles. However, the subtraction procedure has to face the problem of pseudogap formation occurring in the underdoped regime: At substantially high temperatures (above $300 \mathrm{~K}$ ) there is no pseudogap, which instead sets in below $200 \mathrm{~K}$. The anomalous absorption peak we are interested in starts to appear on top of the (pseudogapped) broad absorption spectra at lower $T$. Thus, when the non-pseudogapped spectra at $T \approx 300 \mathrm{~K}$ are subtracted from the lowtemperature pseudogapped ones, a negative differential absorption is found over the frequency range of the pseudogap. Although this is not crucial for the qualitative description of the anomalous peaks, to get quantitatively more precise fits we exploit the fact that the pseudogap sets in rather rapidly and, once established, depends only very weakly on $T$. Therefore, we add a smooth parabolic contribution $\chi_{b}^{\prime \prime}=\omega\left(\Omega_{M A X}-\omega\right) /[B(T)]^{2}$, with $\omega$ in $\mathrm{cm}^{-1}$ and $T$ in $\mathrm{K}$, just designed to cancel the negative part at each temperature. For YBCO we take $\Omega_{M A X}=1000, B(55)=760, B(86)=860$, $B(127)=1100$, while at $T=190,254,282 \mathrm{~K}$ no compensation is needed because the pseudogap is not open. For LSCO at $x=0.02$ we take $\Omega_{M A X}=800, B(35)=500$, $B(88)=500, B(125)=600, B(182)=1000$, while at $T=255 \mathrm{~K}$ again no compensation is needed because the pseudogap is not open. The same procedure is carried out at $x=0.04$, with $\Omega_{M A X}=1000, B(52)=600$, $B(105)=600, B(137)=800, B(154)=1000, B(169)=$ $800, B(207)=1500, B(252)=2000, B(301)=2000$. Fig.9 exemplifies the procedure for LSCO with $x=0.04$ at $T=52 \mathrm{~K}$. The blue curve represents the raw data, from which we subtract the red data at $T=331 \mathrm{~K}$, obtaining the purple curve with unphysical negative absorption. The pseudogap effect is then corrected by the addition of the smooth parabolic contribution, leading to the final black curve. Once these differential spectra are thus brought to have a zero background we proceed to fit the strongly $T$-dependent anomalous peaks.

\section{Appendix C: Pseudogap, Fermi arcs, and Raman response suppression}

The strongly underdoped phase of cuprates is characterized by the presence of a pseudogap that strongly suppresses the low-energy electronic degrees of freedom. In particular, the electronic states in the so-called antinodal regions of the $\mathrm{BZ}$, around $( \pm \pi, 0),(0, \pm \pi)$, are gapped, while the so-called nodal states, along the $( \pm 1, \pm 1)$ directions, survive giving rise to Fermi arcs which shrink upon lowering temperature and doping. In this appendix we investigate the effects of this suppression of the lowenergy electronic states on the coupling between the Raman vertices and the NCMs. Indeed the fermionic loops entering the diagrams of Fig. 1 involve the integration over fermionic degrees of freedom coupled to the nearly critical CMs, with the low-energy fermions being the

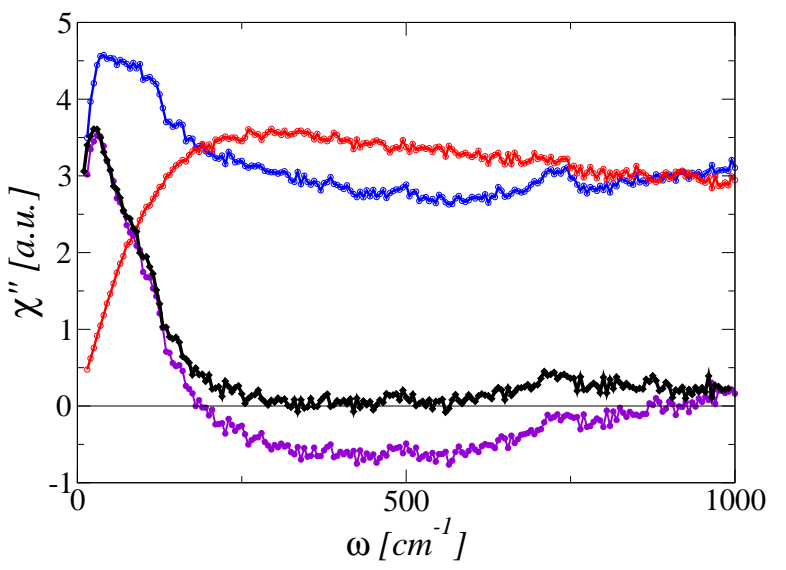

Figure 9. Subtraction procedure on the raw Raman data of a LSCO sample at $x=0.04$ and $T=52 \mathrm{~K}$ (blue curve and symbols). The red curve and symbols correspond to the raw data at $T=331 \mathrm{~K}$. Once the latter are subtracted from the former, the purple curve and symbols are obtained. To eliminate the negative absorption, the parabola $\chi_{b}^{\prime \prime}=\omega(1000-\omega) / 500^{2}$, with $\omega$ in $\mathrm{cm}^{-1}$, is added to finally yield the absorption reported with the black curve and symbols.

most effective in coupling to the low-energy CMs. Therefore the opening of gaps in the electronic spectra naturally entails a substantial reduction of the overall response of the CM. However, the Raman vertices $\gamma_{i}(\mathbf{k})$ weight differently the fermionic states along the Fermi surface and it is therefore quite natural that the fermionic loops are differently suppressed depending on the channel. To estimate this effect is the aim of this appendix. More specifically, we will consider the NCMs only, because the CO modes in the very underdoped LSCO were shown in Ref. [50] to be only visible in the $B_{2 g}$ channel. So it would be meaningless to compare the pseudogap effects in the two Raman channels. On the contrary, the NCM are singular at $\mathbf{q} \approx 0$ and therefore should give a strong contribution to the Raman response in both channels. This appendix will instead demonstrate that the interplay of Raman vertices and momentum dependence of the pseudogap strongly suppress the loop in the $B_{1 g}$ channel in comparison to the $B_{2 g}$ case.

We adopt the simplifying assumption of a circular Fermi surface and we start from the expression for the fermionic loop, Eq. (6). Since the NCMs are singular at small q, we expand in this limit the quantity $\Delta \xi_{\mathbf{k}} \equiv \xi_{\mathbf{k}}-\xi_{\mathbf{k}+\mathbf{q}}$. Expanding up to order $q^{2}$ the denominator and exploiting the $\delta$ function to perform the integral along the radial momentum variable, one obtains

$$
\Lambda_{i} \approx \frac{2 M^{2}}{(2 \pi)^{2}} \int_{0}^{2 \pi} \mathrm{d} \theta F(\theta) \frac{\gamma_{i}(\theta)}{1-\frac{q^{2}}{2 k_{F}^{2}}+\cos (2 \theta-2 \phi)}
$$

where $\phi$ is the angle between $\mathbf{q}$ and the $x$ axis in reciprocal space. At this stage we have phenomenologically 


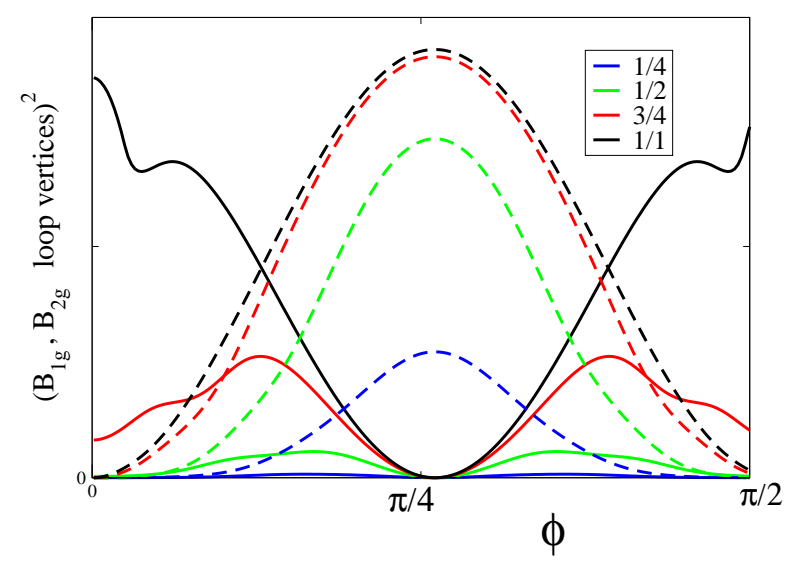

Figure 10. Square of the fermionic loops as a function of the angle $\phi$ calculated according to Eq. C1 in the $B_{1 g}$ channel (solid lines) and in the $B_{2 g}$ channel (dashed lines). The $F(\theta)$ function [Eq. C2 ] has been set to produce arcs shrinking the Fermi surface by a factor $1\left(\Theta_{M}=\pi / 4\right.$, whole Fermi surface, black curves), $0.75\left(\Theta_{M}=3 \pi / 16\right.$, red curves $), 0.5\left(\Theta_{M}=\right.$ $\pi / 8$, green curves), and 0.25 ( $\Theta_{M}=\pi / 16$, blue curves). The parameter $\Delta_{\theta}^{2}=0.05$, was set to smoothen the angular cutoff producing the arcs. The boson momentum $q$ has been chosen such that $q^{2} / 2 k_{F}^{2}=0.01$, while $M=1$. introduced a function

$$
F(\theta)=\sum_{n} \frac{1}{1+e^{\left\{[\theta-(2 n-1) \pi / 4]^{2}-\Theta_{M}^{2}\right\} / \Delta_{\theta}^{2}},}
$$

with $n=1,2,3,4$, which simulates the effect of the pseudogap on the Fermi surface of the 1-4 quadrants. Specifically this function leaves the states near the diagonal untouched, while for $\Theta_{M}<\pi / 4$ it rather sharply suppresses the integration in the gapped antinodal regions for $\theta$ 's far from the nodal direction $\theta=\pi / 4$ (for $\Theta_{M}=\pi / 4$ one recovers the full ungapped Fermi surface). This essentially restricts the integration in Eq. (C1) to the angles of a Fermi arc allowing to explore the different action of the Fermi surface shrinking on the value of the fermionic loop. The parameter $\Delta_{\theta}$ measures how rapidly the pseudogap is switched on and off along the FS, and we take it to be much smaller than $\pi / 4$.

Fig. 10 displays the square of the fermionic loops in the two Raman channels as a function of the angle $\phi$ between the boson transferred momentum $\mathbf{q}$ and the $x$ axis. The calculation clearly shows the increasingly strong suppression of the $B_{1 g}$ fermionic loop (solid curves) upon reducing the length of the Fermi arcs. The suppression is much less pronounced in the $B_{2 g}$ fermionic loop (dashed lines). These results are rather natural because the pseudogap suppresses the states that more effectively contribute to the $B_{1 g}$ loop, while the fermion quasiparticles contributing more to the $B_{2 g}$ channel survive in the Fermi arcs.
1 S. A. Kivelson, I. P. Bindloss, E. Fradkin, V. Oganesyan, J. M. Tranquada, A. Kapitulnik, and C. Howald, Rev. Mod. Phys. 75, 1201 (2003), and references therein.

2 U. Löw, et al., Phys. Rev. Lett. 72, 1918 (1994); Z. Nussinov, et al., Phys. Rev. Lett. 83, 472 (1999); J. Lorenzana, C. Castellani, and C. Di Castro, Phys. Rev. B 64, 235127 (2001); J. Lorenzana, C. Castellani, and C. Di Castro, Europhys. Lett. 57, 704 (2002); R. Jamei, S. Kivelson, and B. Spivak, Phys. Rev. Lett. 94, 056805, (2005); C. Ortix, J. Lorenzana, M. Beccaria, and C. Di Castro, Phys. Rev. B 75, 195107 (2007); C. Ortix, J. Lorenzana, and C. Di Castro, Phys. Rev. Lett. 100, 246402 (2008).

3 M. A. Metlitski and S. Sachdev, Phys. Rev. B 82, 075128 (2010); K. B. Efetov, H. Meier, and C. Pepin, Nat. Phys. 9, 442, (2013); S. Sachdev, and R. La Placa, Phys. Rev. Lett. 111, 027202 (2013).

4 C. Castellani, C. Di Castro, and M. Grilli, Phys. Rev. Lett. 75, 4650 (1995).

5 S. Andergassen, S. Caprara, C. Di Castro, and M. Grilli, Phys. Rev. Lett. 87, 056401 (2001).

6 C. Castellani, C. Di Castro, and M. Grilli, Z. Phys. B 103, 137 (1996).

7 C. Castellani, C. Di Castro, and M. Grilli, J. of Phys. and Chem. of Sol. 59, 1694 (1998).

8 Yuxuan Wang and Andrey Chubukov, arXiv:1401.0712

9 J. M. Tranquada, B. J. Sternlieb, J. D. Axe, Y. Nakamura and S. Uchida, Nature 375, 561 (1995).

10 J. M. Tranquada, J. D. Axe, N. Ichikawa, Y. Nakamura, S.
Uchida, and B. Nachumi, Phys. Rev. B 54, 7489 (1996).

11 J. M. Tranquada, J. D. Axe, N. Ichikawa, A. R. Moodenbaugh, Y. Nakamura, and S. Uchida, Phys. Rev. Lett. 78, 338 (1997).

12 M. Fujita, H. Goka, K. Yamada, and M. Matsuda, Phys. Rev. Lett. 88, 167008 (2002).

13 H.-H. Klauss, W. Wagener, M. Hillberg, W. Kopmann, H. Walf, F. J. Litterst, M. Hücker, and B. Büchner, Phys. Rev. Lett. 85, 4590 (2000).

14 P. Abbamonte, A. Rusydi, S. Smadici, G. D. Gu, G. A. Sawatzky, and D. L. Feng, Nature Phys. 1, 155 (2005).

15 J. Fink, E. Schierle, E. Weschke, J.Geck, D. Hawthorn, V. Soltwisch, H. Wadati, and Hsueh-Hung Wu, Phys. Rev. B 79, 100502 (2009).

16 K. Yamada, C. H. Lee, K. Kurahashi, J. Wada, S. Wakimoto, S. Ueki, H. Kimura, Y. Endoh, S. Hosoya, G. Shirane, R. J. Birgeneau, M. Greven, M. A. Kastner, and Y. J. Kim, Phys. Rev. B 57, 6165 (1998).

17 J. M. Tranquada, H. Woo, T. G. Perring, H. Goka, G. D. Gu, G. Xu, M. Fujita, and K. Yamada, Nature 429, 534 (2004).

18 N. B. Christensen, D. F. McMorrow, H. M. Rönnow, B. Lake, S. M. Hayden, G. Aeppli, T. G. Perring, M. Mangkorntong, M. Nohara, and H. Tagaki, Phys. Rev. Lett. 93, 147002 (2004).

19 S. M. Hayden, H. A. Mook, Pengcheng Dai, T. G. Perring, and F. Dogan, Nature 429, 531 (2004).

${ }^{20}$ V. Hinkov, D. Haug, B. Fauqué, P. Bourges, Y. Sidis, A. 
Ivanov, C. Bernhard, C. T. Lin, and B. Keimer, Science 319, 597 (2008).

21 J. Lorenzana and G. Seibold, Phys. Rev. Lett. 89, 136401 (2002)

22 G. Seibold and J. Lorenzana, Phys. Rev. Lett. 94, 107006 (2005).

${ }^{23}$ G. Seibold and J. Lorenzana, Phys. Rev. B 73, 144515 (2006)

24 N.L. Saini, J. Avila, A. Bianconi, A. Lanzara, M.C. Asensio, S. Tajima, G.D. Gu, N. Koshizuka, Phys. Rev. Lett. 79, 3467 (1997).

25 T. Wu, H. Mayaffre, S. Krämer, M. Horvatić, C. Berthier, W. N. Hardy, R. Liang, D. A. Bonn, and M.-H. Julien, Nature 477, 191 (2011).

26 T. Wu, H. Mayaffre, S. Krämer, M. Horvatić, C. Berthier, P. L. Kuhns, A. P. Reyes, R. Liang, W. N. Hardy, D. A. Bonn, and M.-H. Julien, Nat. Commun. 4, 2113 (2013).

27 C. Howald, H. Eisaki, N. Kaneko, M. Greven, A. Kapitulnik, Phys. Rev. B 67014533 (2003).

28 M. Vershinin, Shashank Misra, S. Ono, Y. Abe, Yoichi Ando, Ali Yazdani, Science 3031995 (2004).

${ }^{29}$ Y. Kohsaka, C. Taylor, K. Fujita, A. Schmidt, C. Lupien, T. Hanaguri, M. Azuma, M. Takano, H. Eisaki, H. Takagi, S. Uchida, J.C. Davis, Science 3151380 (2007).

${ }^{30}$ G. Ghiringhelli, M. Le Tacon, M. Minola, S. BlancoCanosa, C. Mazzoli, N. B. Brookes, G. M. De Luca, A. Frano, D. G. Hawthorn, F. He, T. Loew, M. Moretti Sala, D. C. Peets, M. Salluzzo, E. Schierle, R. Sutarto, G. A. Sawatzky, E. Weschke, B. Keimer, L. Braicovich, Science 337, 821 (2012).

31 J. Chang, E. Blackburn, A. T. Holmes, N. B. Christensen, J. Larsen, J. Mesot, R. Liang, D. A. Bonn, W. N. Hardy, A. Watenphul, M. V. Zimmermann, E. M. Forgan, and S. M. Hayden, Nat. Phys. 8, 871 (2012).

32 R. Comin, R. Sutarto, F. He, E. da Silva Neto, L. Chauviere, A. Frano, R. Liang, W.N. Hardy, D.A. Bonn, Y. Yoshida, H. Eisaki, J. E. Hoffman, B. Keimer, G.A. Sawatzky, and A. Damascelli, arXiv:1402:5415.

33 S. Blanco-Canosa, A. Frano, E. Schierle, J. Porras, T. Loew, M. Minola, M. Bluschke, E. Weschke, B. Keimer, and M. Le Tacon, Phys. Rev. B 90, 054513 (2014).

34 S. Caprara, C. Di Castro, B. Muschler, W. Prestel, R. Hackl, M. Lambacher, A. Erb, S. Komiya, Y. Ando, and M. Grilli, Phys. Rev. B 84 , 054508 (2011).

35 G. Mazza, M. Grilli, C. Di Castro, and S. Caprara, Phys. Rev. B 87, 014511 (2013)

${ }^{36}$ For a review, see, e.g., D. R. Garcia and A. Lanzara, Adv. Cond. Mat. Phys. Volume 2010, Article ID 807412, doi:10.1155/2010/807412, and references therein.

37 A. J. Millis, Phys. Rev. B 81, 035117 (2010).

38 D. Haug, V. Hinkov, Y. Sidis, P. Bourges, N. B. Christensen, A. Ivanov, T. Keller, C. T. Lin, and B. Keimer, New J. Phys. 12, 105006 (2010).

39 R. Daou, J. Chang, David LeBoeuf, Olivier CyrChoinière, Francis Laliberté, Nicolas Doiron-Leyraud, B. J. Ramshaw, R. Liang, D. A. Bonn, W. N. Hardy, and L. Taillefer, Nature (London) 463, 519 (2010).

40 A. Mesaros, K. Fujita, H. Eisaki, S. Uchida, J. C. Davis, S.
Sachdev, J. Zaanen, M. J. Lawler, and E.-A. Kim, Science 333, 426 (2011).

${ }^{41}$ M. J. Lawler, K. Fujita, J. Lee, A. R. Schmidt, Y. Kohsaka, C. K. Kim, H. Eisaki, S. Uchida, J. C. Davis, J. P. Sethna, et al., Nature 466, 347 (2010).

42 S. A. Kivelson, E. Fradkin, and V. J. Emery, Nature (London) 393, 550 (1998).

43 M. Vojta, Adv. Phys. 58, 699 (2009).

44 K. Sun, M. J. Lawler, and E.-A. Kim, Phys. Rev. Lett. 104, 106405 (2010).

45 G. Seibold, M. Capati, C. Di Castro, M. Grilli, and J. Lorenzana, Phys. Rev. B 87, 035138 (2013).

46 S. Wakimoto, R. J. Birgeneau, M. A. Kastner, Y. S. Lee, R. Erwin, P. M. Gehring, S. H. Lee, M. Fujita, K. Yamada, Y. Endoh, K. Hirota, and G. Shirane, Phys. Rev. B 61, 3699 (2000).

47 M. Capati, S. Caprara, C. Di Castro, M. Grilli, G. Seibold, and J. Lorenzana, unpublished.

48 S. Shastry and B. Shraiman, Phys. Rev. Lett. 103, 1078 (1990).

49 T. P. Devereaux and R. Hackl, Rev. Mod. Phys. 79, 175 (2007).

50 S. Caprara, C. Di Castro, M. Grilli, and D. Suppa, Phys. Rev. Lett. 95, 117004 (2005).

51 S. Caprara, M. Grilli, C. Di Castro, and T. Enss, Phys. Rev. B 75, 140505(R) (2007).

52 L. Tassini et al., Phys. Rev. B 78, 020511 (2008).

53 S. Caprara, C. Di Castro, T. Enss, and M. Grilli, J. Phys. Chem. Sol. 69, 2155 (2008).

54 M. Grilli, S. Caprara, C. Di Castro, T. Enss, R. Hackl, B. Muschler, and W. Prestel, Physica B 404, 3070 (2009).

55 B Muschler, W Prestel, E Schachinger, J P Carbotte, R Hackl, Shimpei Ono and Yoichi Ando, J. Phys.: Condens. Matter 22375702 (2010)

${ }^{56}$ L. Tassini, F. Venturini, Q.-M. Zhang, R. Hackl, N. Kikugawa, and T. Fujita, Phys. Rev. Lett. 95, 117002 (2005).

57 T. Yoshida, X. J. Zhou, T. Sasagawa, W. L. Yang, P.V. Bogdanov, A. Lanzara, Z. Hussain, T. Mizokawa, A. Fujimori, H. Eisaki, Z.-X. Shen, T. Kakeshita, and S. Uchida, Phys. Rev. Lett. 91, 027001 (2003)

58 X. J. Zhou, T. Yoshida, D.-H. Lee, W. L. Yang, V. Brouet, F. Zhou, W. X. Ti, J.W. Xiong, Z. X. Zhao, T. Sasagawa, T. Kakeshita, H. Eisaki, S. Uchida, A. Fujimori, Z. Hussain, and Z.-X. Shen, Phys. Rev. Lett. 92, 187001 (2004).

59 Y. Ando, A. N. Lavrov, S. Komiya, K. Segawa, and X. F. Sun, Phys. Rev. Lett. 87, 017001 (2001).

60 Yoichi Ando, Y. Kurita, Seiki Komiya, S. Ono, and Kouji Segawa, Phys. Rev. Lett. 92, 197001 (2004).

61 V. Oganesyan, S. A. Kivelson, and E. Fradkin, Phys. Rev. B 64, 195109 (2001).

62 M. Garst and A. V. Chubukov, Phys. Rev. B 81, 235105 (2010).

63 A. J. Millis, H. Monien, and D. Pines, Phys. Rev. B 42, 167 (1990).

64 Ar. Abanov, A. Chubukov, and J. Schmalian, Adv. Phys. 52, 119 (2003), and references therein.

65 M. Zacharias, P. Wölfle, and M. Garst, Phys. Rev. B 80, 165116 (2009). 